\title{
Diagnosis and management of acute kidney injury in patients with cirrhosis: revised consensus recommendations of the International Club of Ascites
}

\author{
Paolo Angeli, ${ }^{1}$ Pere Gines, ${ }^{2,3,4,5}$ Florence Wong, ${ }^{6}$ Mauro Bernardi, ${ }^{7}$ \\ Thomas D Boyer, ${ }^{8}$ Alexander Gerbes, ${ }^{9}$ Richard Moreau, ${ }^{10,11,12}$ \\ Rajiv Jalan, ${ }^{13}$ Shiv K Sarin, ${ }^{14}$ Salvatore Piano, ${ }^{1}$ Kevin Moore, ${ }^{15}$ \\ Samuel S Lee, ${ }^{16}$ Francois Durand, ${ }^{17,18}$ Francesco Salerno, ${ }^{19}$ \\ Paolo Caraceni, ${ }^{7}$ W Ray Kim, ${ }^{20}$ Vicente Arroyo, ${ }^{2,3,4}$ \\ Guadalupe Garcia-Tsao ${ }^{21}$
}

\section{INTRODUCTION}

Acute renal failure (ARF) is a common complication in patients with decompensated cirrhosis. The traditional diagnostic criteria of renal failure in these patients were proposed in $1996^{1}$ and have been refined in subsequent years. ${ }^{2}$ According to these criteria, ARF is defined as an increase

\footnotetext{
${ }^{1}$ Unit of Hepatic Emergencies and Liver Transplantation, Department of Medicine-DIMED, University of Padova, Padova, Italy; ${ }^{2}$ Liver Unit, Hospital Clinic, University of Barcelona, Barcelona, Spain; ${ }^{3}$ Institut $\mathrm{d}^{\prime}$ Investigacions Biomediques Agust Pi i Sunyer (IDIBAPS), Barcelona, Spain; ${ }^{4}$ Centro de Investigación Biomédica en Red en Enfermedades Hepáticas y Digestivas (CIBEREHD), Barcelona, Spain; ${ }^{5}$ Instituto Reina Sofia d'Investigación en Nefrologia (IRSIN), Barcelona, Spain; ${ }^{6}$ Division of Gastroenterology, Department of Medicine, University of Toronto, Toronto, Canada; ${ }^{7}$ Dipartimento di Scienze Mediche e Chirurgiche, Alma Mater Studiorum, University of Bologna, Bologna, Italy; ${ }^{8}$ Department of Medicine, Liver Research Institute, University of Arizona, College of Medicine, Tucson, Arizona, USA; ${ }^{9}$ Liver Unit, Klinikum Munich, Ludwig Maximilian University of Munich, Munich, Germany; ${ }^{10}$ Inserm U1149, Centre de recherche sur I'Inflammation (CRI), Paris, France; ${ }^{11}$ UMR S 1149, Université Paris Diderot, Paris, France: ${ }^{12}$ DHU UNITY, Service d'hépatologie, Hôpital Beaujon, APHP, Clichy, France; ${ }^{13}$ Liver Failure Group, UCL Institute for Liver and Digestive Health, UCL Medical School, Royal Free Hospital, London, UK; ${ }^{14}$ Department of Hepatology, Institute of Liver and Biliary Sciences, New Delhi, India; ${ }^{15}$ UCL Institute of Liver and Digestive Health, Royal Free Campus, University College London, London, UK; ${ }^{16}$ Liver Unit, University of Calgary, Calgary, Canada; ${ }^{17}$ Hepatology and Liver Intensive Care Unit, Hospital Beaujon, Clichy, France; ${ }^{18}$ INSERM U773, Centre de Recherche Biomédicale Bichat Beaujon CRB3, Clichy, France; ${ }^{19}$ Policlinico IRCCS San Donato, Medicina Interna ed Epatologia, Università di Milano, Milan, Italy; ${ }^{20}$ Division of Gastroenterology and Hepatology, Stanford University Medical School, Palo Alto, California, USA; ${ }^{21}$ Division of Digestive Diseases, Yale University School of Medicine, New Haven, Connecticut, USA
}

Correspondence to Professor Paolo Angeli, Department of Medicine (DIMED) and Unit of Hepatic Emergencies and Liver Transplantation, University of Padova, Via Giustiniani 2, Padova 35100, Italy; pangeli@unipd.it in serum creatinine $(\mathrm{sCr})$ of $\geq 50 \%$ from baseline to a final value $>1.5 \mathrm{mg} / \mathrm{dL}$ $(133 \mu \mathrm{mol} / \mathrm{L})$. However, the threshold value of $1.5 \mathrm{mg} / \mathrm{dL}(133 \mu \mathrm{mol} / \mathrm{L}) \mathrm{sCr}$ to define renal failure in patients with decompensated cirrhosis has been challenged. ${ }^{3} 4$ In addition, the timeframe to distinguish acute from chronic renal failure has not been clearly identified, the only exception being type 1 hepatorenal syndrome (HRS). Meanwhile, new definitions for ARF, now termed acute kidney injury (AKI), have been proposed and validated in patients without cirrhosis. ${ }^{5-7}$ Recently these new criteria were also proposed and applied in the diagnosis of AKI in patients with cirrhosis. ${ }^{3}$ 8-15 Thus, in December 2012, the International Club of Ascites (ICA) organised a consensus development meeting in Venice, Italy, in order to reach a new definition of AKI in patients with cirrhosis. The discussion among the experts continued thereafter for 2 years, both online and through several meetings, between those experts who had different positions on crucial points on the subject. This paper reports the scientific evidence supporting the final proposal of a new approach to the diagnosis and treatment of this condition, on which the experts agreed.

\section{DIAGNOSTIC CRITERIA OF AKI AND THEIR APPLICATION IN PATIENTS WITH CIRRHOSIS}

AKI is defined as an acute significant reduction in the glomerular filtration rate (GFR). sCr remains the most practical biomarker of renal function in patients with ARF (with or without cirrhosis). However, sCr as a biomarker of renal function has many limitations in clinical practice since it is influenced by bodyweight, race, age, and gender. The use of $\mathrm{sCr}$ in patients with cirrhosis is also affected by: (1) decreased formation of creatinine from creatine in muscles, secondary to muscle wasting; ${ }^{16}$ (2) increased renal tubular secretion of creatinine; ${ }^{17}$ (3) the increased volume of distribution in cirrhosis that may dilute sCr; (4) interference with assays for sCr by elevated bilirubin. ${ }^{18}$ As a consequence, measurement of $\mathrm{sCr}$ in patients with cirrhosis overestimates GFR or kidney function. Therefore, the use of a fixed threshold of $\mathrm{sCr}$ at $1.5 \mathrm{mg} / \mathrm{dL}(133 \mu \mathrm{mol} / \mathrm{L})$ to define AKI in cirrhosis $^{12}$ is problematic, because of two crucial problems. The first is that an $\mathrm{sCr}$ value of $1.5 \mathrm{mg} / \mathrm{dL}(133 \mu \mathrm{mol} / \mathrm{L})$ often signifies that GFR is markedly decreased (to $\sim 30 \mathrm{~mL} / \mathrm{min}$ ); $;^{19}$ secondly, the fixed threshold does not take into account the dynamic changes in $\mathrm{sCr}$ that occur in the preceding days or weeks, which are needed to distinguish between acute and chronic kidney injury. Since the use of a single value of $\mathrm{sCr}$ is not sufficient to diagnose AKI, a dynamic definition referring to an acute increase of $\mathrm{sCr}$ to $\geq 50 \%$ from baseline to a final value $\geq 1.5 \mathrm{mg} / \mathrm{dL}$ $(133 \mu \mathrm{mol} / \mathrm{L})$ has been used in several clinical studies in patients with cirrhosis (table 1). AKI, as defined by these criteria, was a strong predictor of in-hospital mortality in patients with cirrhosis. ${ }^{20-23}$

In recent years, diagnostic criteria have been proposed for the diagnosis of ARF in non-cirrhotic patients, now termed AKI. In particular, two separate bodies developed and published two consensus definitions of AKI: the Acute Dialysis Quality Initiative group for the Risk, Injury, Failure, Loss of Renal Function and End-Stage Renal Disease (RIFLE) criteria; and the Acute Kidney Injury Network (AKIN) group for the AKIN criteria (table 1). ${ }^{5}$ More recently, a panel of experts has suggested combining part of the AKIN criteria (increase of $\mathrm{sCr}$ of $0.3 \mathrm{mg} / \mathrm{dL}(26.5 \mu \mathrm{mol} / \mathrm{L})$ within $48 \mathrm{~h}$ or by $\geq 50 \%$ from baseline together with a reduction in urine output to $<0.5 \mathrm{~mL} / \mathrm{kg} / \mathrm{h}$ for $>6 \mathrm{~h}$ ) with part of the RIFLE criteria (increase of $\mathrm{sCr} \geq 50 \%$ within 1 week or a reduction in GFR by $>25 \%$ together with a reduction in urine output to $<0.5 \mathrm{~mL} /$ $\mathrm{kg} / \mathrm{h}$ for $>6 \mathrm{~h}$ ), thus leading to the proposal of the Kidney Disease Improving Global Outcome (KDIGO) criteria ${ }^{7}$ (table 1). However, the use of a reduction of urine output in patients with cirrhosis and ascites as a diagnostic criterion is a problem, since these patients are frequently oliguric with avid sodium retention and yet may maintain a relatively normal GFR. ${ }^{24}$ Conversely, these patients may have an increased urine output because of diuretic treatment. Thus, urine collection is often inaccurate in clinical 


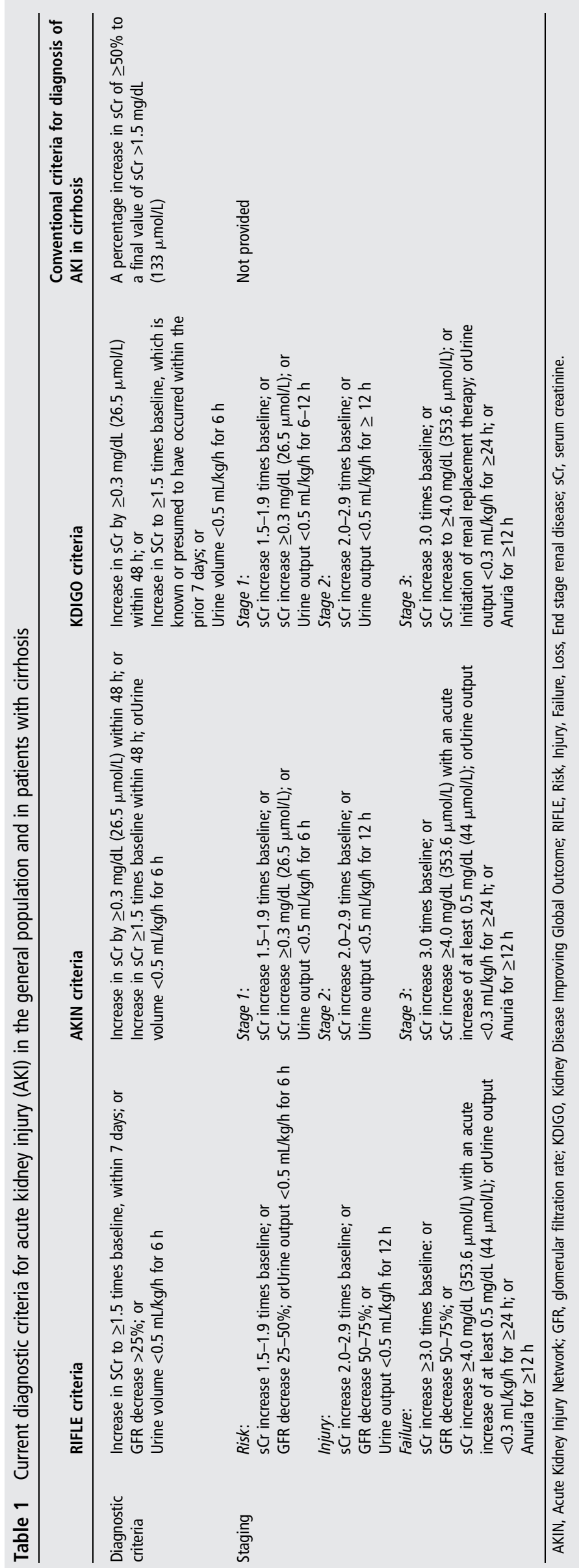

practice and the use of kinetic changes in sCr becomes the crux of the definition for the diagnosis of AKI in cirrhosis.

The main differences between these new criteria over the conventional criteria in patients with cirrhosis are the following: (1) an absolute increase in $\mathrm{sCr}$ is considered; (2) the threshold of $\mathrm{sCr}$ $\geq 1.5 \mathrm{mg} / \mathrm{dL}(133 \mu \mathrm{mol} / \mathrm{L})$ is abandoned; and (3) a staging system of AKI, based on a change in $\mathrm{sCr}$ over a slightly longer time frame, arbitrarily set at 1 week to enable assessment for progression of stage (modified from AKIN staging) as well as a regression of stage (table 1). AKIN criteria have been shown to be a good predictor of mortality in large cohorts of hospitalised cirrhotic patients, including those in intensive care units ${ }^{25}$ and the critically ill. ${ }^{26}$ More recently, AKI as diagnosed with AKIN criteria has been shown to be associated with increased mortality in patients with cirrhosis who were hospitalised in regular wards in an AKIN stagedependent fashion. ${ }^{8-13} 15$ Further, the progression of AKI through stages (eg, from stage 1 to 2 or stage 2 to 3 ) was strongly correlated with an increased mortality in these patients. ${ }^{8-10}$ Nevertheless, a comparison of the prognostic accuracy of the conventional criteria and the new criteria in patients with cirrhosis was considered crucial for the development of a new algorithm for the management of AKI and was proposed by the ICA in 2011 . $^{3}$

However, the cut-off value of $1.5 \mathrm{mg} / \mathrm{dL}$ $(133 \mu \mathrm{mol} / \mathrm{L})$ still has important resonance with many clinicians. Two prospective studies have recently shown that a cut-off value of sCr of $1.5 \mathrm{mg} / \mathrm{dL}(133$ $\mu \mathrm{mol} / \mathrm{L}$ ) is useful to predict progression of AKI and consequently the prognosis in patients with cirrhosis. ${ }^{9} 10$ Thus, an $\mathrm{sCr}$ $\geq 1.5 \mathrm{mg} / \mathrm{dL} \quad(133 \mu \mathrm{mol} / \mathrm{L})$ was the only predictive factor for progression of the initial AKI stage (AKI stage at the first fulfilment of AKIN criteria) to a higher AKI stage during hospitalisation (peak AKI stage). Thereafter, it was also shown that the cut-off value of $\mathrm{sCr} \geq 1.5 \mathrm{mg} / \mathrm{dL}$ $(133 \mu \mathrm{mol} / \mathrm{L})$ was important when patients with peak AKI stage 1 were considered. In fact, patients with AKI stage 1 could be divided into two groups: those whose peak sCr did not exceed $1.5 \mathrm{mg} / \mathrm{dL}$ (stage 1-A), whose short term mortality might be similar to those without AKI and in whom regression might occur more frequently; ${ }^{9}{ }^{10}$ and those whose peak $\mathrm{sCr}$ exceeded $1.5 \mathrm{mg} / \mathrm{dL}$ (stage 1-B), whose short term mortality was higher than those without AKI. ${ }^{9} 10$ Patients with AKI stage 2 and 3 have the highest mortality. ${ }^{8-10}$ However, whether these observations can 
be generalised to all hospitalised patients with cirrhosis should be assessed in future studies. In fact, as far as the impact of peak AKI stage 1 on in-hospital mortality, it has recently been observed that in patients who developed AKI as a consequence of a bacterial infection, those with stage $1 \mathrm{AKI}$ and a final $\mathrm{sCr} \leq 1.5 \mathrm{mg} / \mathrm{dL}(133 \mu \mathrm{mol} / \mathrm{L})$ had a higher short term mortality compared to those without AKI. ${ }^{13} 27$ In addition, regarding regression of AKI stage, it has recently been observed (in nonhospitalised patients) that despite resolution of most AKI episodes in patients with advanced cirrhosis, a gradual and significant increase in $\mathrm{sCr}$ and a gradual reduction in mean arterial pressure were observed during follow-up, associated with a significant reduction in mid-term survival compared with non-AKI patients. ${ }^{11}$ Indeed, the main lesson learnt from the application of AKIN criteria is that even a small increase in $\mathrm{sCr}$ should be identified as early as possible for potential early interventions.

\section{WHY DO WE NEED TO CHANGE THE CONVENTIONAL DIAGNOSTIC CRITERIA FOR AKI?}

A recent editorial on the topic of AKI in cirrhosis $^{28}$ asked the question: "Should we change current definition and diagnostic criteria of renal failure in cirrhosis?" Currently, studies on AKI in patients with cirrhosis showed that AKI defined by an absolute increase in $\mathrm{sCr} \geq 0.3 \mathrm{mg} / \mathrm{dL}$ (26.5 $\mu \mathrm{mol} / \mathrm{l})$ and/or $\geq 50 \%$ from baseline is associated with a higher probability of the patients being transferred to the intensive care unit, a longer hospital stay, and an increased in-hospital as well as 90-day and mid-term mortality. ${ }^{8-15}$ On the basis of this evidence, all the experts agreed that it was time to change our current definition of renal failure by introducing a modified version of the KDIGO criteria for the diagnosis of AKI in patients with cirrhosis (table 2). In the new ICA criteria for the diagnosis of AKI, the use of urine output as one of the criteria has been removed since it does not apply to patients with cirrhosis (ie, many patients are oliguric but have preserved kidney function) and it has never been investigated. Further, two other changes to the KDIGO criteria were adopted, namely: (1) a sCr within the last 3 months before admission is considered a baseline value for the diagnosis of AKI when a value within the previous 7 days is not available; and (2) the calculation of the baseline $\mathrm{sCr}$ by the reverse application of the Modification of Diet in Renal Disease (MDRD) formula, using an arbitrarily defined normal value of GFR of $75 \mathrm{~mL} /$ $\min / 1.73 \mathrm{~m}^{2}$, was not included. These two points are specifically discussed in the next section.

\section{DEFINITION OF BASELINE SERUM CREATININE FOR THE DIAGNOSIS OF AKI}

The first step in applying the ICA-AKI criteria is to define a baseline sCr. It has been stated that a renal disease process that results in a change in $\mathrm{sCr}$ over several weeks cannot be defined as AKI, although it may still represent an important clinical entity. ${ }^{7}$ Nevertheless, as with any clinical scenario, the timeframe for the definition of AKI is somewhat arbitrary, and it is mainly suitable for the diagnosis of AKI in hospitalised patients using a $\mathrm{sCr}$ value on or after admission as baseline (hospitalacquired AKI). However, as in the general population, many patients with cirrhosis can develop AKI before admission to hospital (community-acquired AKI). Indeed, in previous studies where pre-admission values of sCr were used as baseline, the rate of AKI was higher than in those based on $\mathrm{sCr}$ on admission as baseline $(47 \%$ vs $26 \%){ }^{9} \quad 10$ Thus, the diagnosis of community-acquired AKI on admission is related to two possible scenarios: (1) the patient with an available sCr value before admission; and (2) the patient without an $\mathrm{sCr}$ value before admission. The use of pre-admission values of $\mathrm{sCr}$ poses a great dilemma: how far back can a baseline value of $\mathrm{sCr}$ be retrieved and still be expected to be 'valid' for the definition of $\mathrm{AKI}$ ? In the general population, it is reasonable to assume that $\mathrm{sCr}$ will be stable over several months or even years, so that an sCr obtained 6 months or even 1 year previously would reasonably reflect the patient's premorbid baseline. ${ }^{7} 29$ In patients with cirrhosis, an application of a more rigorous time frame for the definition of AKI seems even more important. In fact, in these patients, impairment of renal function may progress gradually as they go from a compensated to a decompensated state and then more rapidly as the decompensated state worsens. In addition, it should be considered that almost all patients with cirrhosis and ascites receive diuretics that can transiently impair renal function and, thus, increase sCr.

Furthermore, it is important to emphasise the variability in $\mathrm{sCr}$ measurements from laboratory to laboratory or even within the same laboratory due to, for example, fluctuations in serum bilirubin in patients with cirrhosis. ${ }^{30} \mathrm{~A}$ sCr obtained $<7$ days before admission would be the ideal condition to use the ICA-AKI criteria, but this timeframe seems unfeasible in most cases. Thus, taking into account the previous experiences, we conclude that use of the last value of sCr within the last 3 months before admission seems

Table 2 International Club of Ascites (ICA-AKI) new definitions for the diagnosis and management of AKI in patients with cirrhosis

\begin{tabular}{|c|c|c|c|}
\hline Subject & \multicolumn{3}{|l|}{ Definition } \\
\hline Baseline $\mathrm{sCr}$ & \multicolumn{3}{|c|}{$\begin{array}{l}\text { A value of } \mathrm{sCr} \text { obtained in the previous } 3 \text { months, when available, can be used as baseline } \mathrm{sCr} \text {. In patients with more than one value within } \\
\text { the previous } 3 \text { months, the value closest to the admission time to the hospital should be used } \\
\text { In patients without a previous } \mathrm{s} C r \text { value, the } \mathrm{sCr} \text { on admission should be used as baseline }\end{array}$} \\
\hline Definition of AKI & \multicolumn{3}{|c|}{$\begin{array}{l}\text { Increase in } \mathrm{sCr} \geq 0.3 \mathrm{mg} / \mathrm{dL} \text { ( } \geq 26.5 \mu \mathrm{mol} / \mathrm{L} \text { ) within } 48 \mathrm{~h} \text {; ora percentage increase } \mathrm{sCr} \geq 50 \% \text { from baseline which is known, or presumed, to } \\
\text { have occurred within the prior } 7 \text { days }\end{array}$} \\
\hline Staging of AKI & \multicolumn{3}{|c|}{$\begin{array}{l}\text { Stage 1: increase in } \mathrm{sCr} \geq 0.3 \mathrm{mg} / \mathrm{dL}(26.5 \mu \mathrm{mol} / \mathrm{L}) \text { or an increase in } \mathrm{sCr} \geq 1.5 \text {-fold to twofold from baseline } \\
\text { Stage 2: increase in } \mathrm{sCr}>\text { two to threefold from baseline } \\
\text { Stage 3: increase of } \mathrm{sCr}>\text { threefold from baseline or } \mathrm{sCr} \geq 4.0 \mathrm{mg} / \mathrm{dL}(353.6 \mu \mathrm{mol} / \mathrm{L}) \text { with an acute increase } \geq 0.3 \mathrm{mg} / \mathrm{dL}(26.5 \mu \mathrm{mol} / \mathrm{L}) \text { or } \\
\text { initiation of renal replacement therapy }\end{array}$} \\
\hline Progression of AKI & \multicolumn{2}{|c|}{$\begin{array}{l}\text { Progression } \\
\text { Progression of AKI to a higher stage and/or need for RRT }\end{array}$} & $\begin{array}{l}\text { Regression } \\
\text { Regression of AKI to a lower stage }\end{array}$ \\
\hline Response to treatment & $\begin{array}{l}\text { No response } \\
\text { No regression of } A K I\end{array}$ & $\begin{array}{l}\text { Partial response } \\
\text { Regression of AKI stage with a reduction of } \mathrm{sCr} \text { to } \geq 0.3 \mathrm{mg} / \mathrm{dL} \\
(26.5 \mu \mathrm{mol} / \mathrm{L}) \text { above the baseline value }\end{array}$ & $\begin{array}{l}\text { Full response } \\
\text { Return of } \mathrm{sCr} \text { to a value within } 0.3 \mathrm{mg} / \mathrm{dL} \\
(26.5 \mu \mathrm{mol} / \mathrm{L}) \text { of the baseline value }\end{array}$ \\
\hline
\end{tabular}


more feasible. ${ }^{10} 13$ In this scenario, a community-acquired AKI may be diagnosed in the case of an increase in $\mathrm{sCr}$ $\geq 50 \%$ from the last $\mathrm{sCr}$ value (table 2). For patients without an available $\mathrm{sCr}$ before hospitalisation, the use of an estimated value of $\mathrm{sCr}$ as the baseline, calculated by the reverse application of the MDRD formula using a predetermined value of GFR $(75 \mathrm{~mL} / \mathrm{min})$, has been suggested for the general population of patients. $^{7}$ However, it is well known that the MDRD formula is inaccurate in the estimation of GFR in patients with cirrhosis, particularly in those with ascites. ${ }^{31}$ As a result, its reverse application in these patients may only add further biases. Preliminary data from the Padua centre suggest that a diagnosis of AKI based on an computed value of $\mathrm{sCr}$ as baseline identifies $<25 \%$ of patients with a measured GFR $<60 \mathrm{~mL} / \mathrm{min}$ on admission (Angeli P et al, unpublished observations). However, among patients without an $\mathrm{sCr}$ value before admission, one scenario deserves specific mention, and that is the case of the patient with an $\mathrm{sCr} \geq 1.5 \mathrm{mg} /$ $\mathrm{dL}(133 \mu \mathrm{mol} / \mathrm{L})$ at admission. The management of such a patient should be based not only on a formal definition of AKI, but also on clinical judgment. Therefore, in a patient with impairment of renal function and a clearly identifiable precipitating event, it would be reasonable to assume that the renal failure represents AKI. Alternatively, the initial sCr may be used as the baseline value, and if AKI criteria are met subsequently then the patient has AKI. This approach was commonly used previously for the diagnosis of type 1 HRS. $^{32}$

\section{A NEW ALGORITHM FOR THE MANAGEMENT OF AKI IN PATIENTS WITH CIRRHOSIS}

According to the new ICA-AKI diagnostic criteria for AKI, we propose a new algorithm for the management of AKI in patients with cirrhosis (figure 1). The algorithm is based on the new staging of AKI.

We recommend that patients with cirrhosis and ascites with initial ICA-AKI stage 1 should be managed as soon as possible with the following measures:

1. Review drug chart: review of all medications (including over-the-counter (OTC) drugs), reduction or withdrawal of diuretic therapy, withdrawal of all potentially nephrotoxic drugs, vasodilators or non-steroidal antiinflammatory drugs (NSAIDs)

2. Plasma volume expansion in patients with clinically suspected hypovolaemia (with crystalloids or albumin or blood (in patients who had AKI as a result of gastrointestinal bleeding) according to clinical judgment)

3. Prompt recognition and early treatment of bacterial infections when diagnosed or strongly suspected.

Patients who respond with a return of $\mathrm{sCr}$ to a value within $0.3 \mathrm{mg} / \mathrm{dL}$ $(26.5 \mu \mathrm{mol} / \mathrm{L})$ of the baseline value should be followed closely (assessment of sCr every 2-4 days during the hospitalisation and checked as outpatients at least every 2-4 weeks during the first 6 months after the discharge) for early identification of potential new episodes of AKI. ${ }^{11}$ In those cases where there is progression of the AKI stage, the patients should be treated as patients who present with ICA-AKI stage 2 and 3. This treatment should include the withdrawal of diuretics, if this had not been previously implemented, as well as the expansion of plasma volume with intravenous albumin at the dose of $1 \mathrm{~g}$ per $\mathrm{kg}$ bodyweight per day for two consecutive days, in order to treat pre-renal AKI and to allow differential diagnosis of AKI (box 1). The maximal dose per day of albumin should not exceed $100 \mathrm{~g}$ as previously suggested. ${ }^{2}$ Further management of patients who do not respond to diuretic withdrawal and plasma volume expansion will obviously

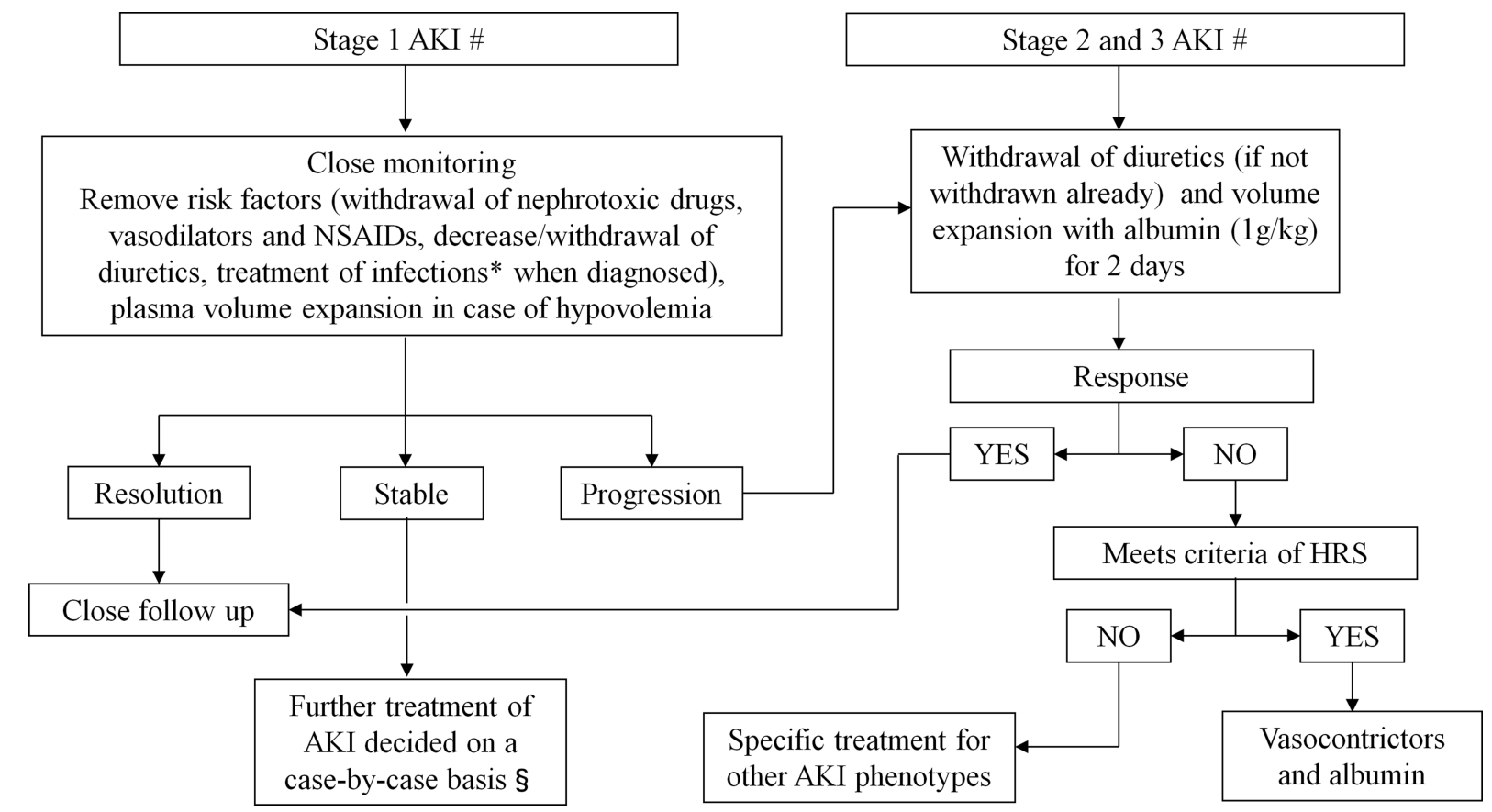

Figure 1 Proposed algorithm for the management of acute kidney injury (AKI) according to International Club of Ascites-AKI (ICA-AKI) classification that combines Kidney Disease Improving Global Outcomes (KDIGO) criteria and conventional criteria in patients with cirrhosis and ascites. Most of the experts had concerns about the use of vasoconstrictors in patients with AKI stage 1 and $\mathrm{sCr}<1.5 \mathrm{mg} / \mathrm{dL}$. For the definition of close follow-up, and/or case-by-case, see the text. *Treatment of spontaneous bacterial peritonitis should include albumin infusion according to current guidelines. \#Initial AKI stage is defined as AKI stage at the time of first fulfilment of the AKI criteria. §No global consensus was reached on this point. HRS, hepatorenal syndrome; NSAIDs, non-steroidal anti-inflammatory drugs; sCr, serum creatinine. 
Box 1 Diagnostic criteria of hepatorenal syndrome (HRS) type of acute kidney injury (AKI) in patients with cirrhosis

\section{HRS-AKI}

- Diagnosis of cirrhosis and ascites

- Diagnosis of AKI according to ICA-AKI criteria

- No response after 2 consecutive days of diuretic withdrawal and plasma volume expansion with albumin $1 \mathrm{~g} / \mathrm{kg}$ bodyweight

- Absence of shock

- No current or recent use of nephrotoxic drugs (NSAIDs, aminoglycosides, iodinated contrast media, etc)

- No macroscopic signs of structural kidney injury ${ }^{*}$, defined as:

- absence of proteinuria ( $>500 \mathrm{mg} /$ day)

- absence of microhaematuria (>50 RBCs per high power field)

- normal findings on renal ultrasonography

*Patients who fulfil these criteria may still have structural damage such as tubular damage. Urine biomarkers will become an important element in making a more accurate differential diagnosis between HRS and acute tubular necrosis.

ICA, International Club of Ascites; NSAIDs, non-steroidal anti-inflammatory drugs; RBCs, red blood cells.

depend on the final diagnosis of the AKI type and, pragmatically, on the differential diagnosis between an HRS-AKI, an intrinsic AKI, and post-renal-AKI (box 1). Thus, another major contribution of this new algorithm is to accelerate the differential diagnostic process among the different types of AKI. However, it should be highlighted that several steps of this algorithm are not based on evidence but just on experts' opinion, and that it should be validated in future prospective clinical studies. In particular, in patients with AKI stage 1 who do not respond but who do not progress to a higher stage, no consensus was obtained among the experts on the specific treatment. All experts agreed to treat these patients according to the right side of the algorithm when the final value of $\mathrm{sCr}$ is $\geq 1.5 \mathrm{mg} / \mathrm{dL}(133 \mu \mathrm{mol} / \mathrm{L})$. Some experts favour the treatment of patients with AKI stage 1 and $\mathrm{sCr}$ $<1.5 \mathrm{mg} / \mathrm{dL}(133 \mu \mathrm{mol} / \mathrm{L})$ in the same way. However, most of the experts did not agree on this because they had concerns about the early use of vasoconstrictors (terlipressin or norepinephrine or midodrine plus octreotide) in these patients in case of HRS-AKI. Thus, further clinical controlled studies are needed to address this relevant issue. In the meantime, decisions about the treatment of these patients should be taken on a case-by-case basis evaluating the aetiology of AKI, the presence or absence of precipitating factors, other organ failures, or comorbid conditions that may contraindicate treatment.

\section{WHY DO WE NEED TO CHANGE THE DIAGNOSTIC CRITERIA OF HRS IN THE SETTING OF AKI?}

A major critical point in the management of AKI in patients with decompensated cirrhosis is whether the diagnostic criteria of type 1 HRS should be revised in light of the new definitions of AKI. The current criteria include a time interval (2 weeks) over which sCr must double to a value $>2.5 \mathrm{mg} / \mathrm{dL}$ for the diagnosis of type 1 HRS. $^{12}$ A revision of these criteria is needed because the current definition of type 1 HRS does not allow physicians to initiate potentially effective treatment, specifically vasoconstrictors and albumin, until the $\mathrm{sCr}$ increases to $\geq 2.5 \mathrm{mg} / \mathrm{dL}$. Since it has been observed that in patients with type $1 \mathrm{HRS}$, a higher $\mathrm{sCr}$ at the beginning of treatment leads to a lower probability of response to terlipressin and albumin, the most investigated and effective treatment of type 1 HRS, ${ }^{33} 34$ it seems prudent not to wait until the $\mathrm{sCr}$ increases beyond $2.5 \mathrm{mg} / \mathrm{dL}$ before starting the treatment. According to the new proposed algorithm, when AKI is characterised by an initial ICA-AKI stage 2 or 3 or by progression of the initial stage despite general therapeutic measures, patients who meet all other diagnostic criteria of HRS provided by the previous definition ${ }^{2}$ should receive vasoconstrictors and albumin, irrespective of the final value of sCr. This makes it possible to remove a barrier to the achievement of a pharmacological response that was linked to the rigid $\mathrm{sCr}$ cut-off value of $>2.5 \mathrm{mg} / \mathrm{dL}$ in the definition of type 1 HRS. The potential advantage of the algorithm is that its application may allow earlier treatment of patients with type $1 \mathrm{HRS}$, leading to a better outcome as compared with the current approach. However, we lack studies where vasoconstrictors were used in the treatment of HRS with lower values of sCr, and caution should be exercised in the use of vasoconstrictors in these patients pending further controlled trials.

Nevertheless, all the experts agreed on the removal of a fixed cut-off value of $\mathrm{sCr}$ from the diagnostic criteria of HRS. This is the only change that they wanted to introduce in the current diagnostic criteria for HRS. As a consequence, all the remaining criteria are maintained (box 1). However, these criteria do not rule out the possibility of renal parenchymal damage. ${ }^{35}$ Thus, all the experts agreed on the potential role of new urinary biomarkers in the differential diagnosis of the different types of AKI in patients with cirrhosis. Several urinary biomarkers of tubular damage, such as neutrophil gelatinase-associated lipocalin (NGAL), kidney injury molecule-1 (KIM-1), interleukin-18 (IL-18), and liver fatty acid-binding protein (L-FABP), have been discovered in recent years. Preliminary experiences from Europe and the USA showed that the use of $\mathrm{NGAL}^{36}$ and/or the combination of urinary biomarkers (NGAL, KIM-1, IL-18, L-FABP and albuminuria) ${ }^{37}$ may be useful in the differential diagnosis of AKI in patients with cirrhosis. These findings need to be confirmed in future studies.

The removal of a fixed cut-off value of sCr from the diagnostic criteria of HRS in the setting of AKI has important implications in the management of these patients. Thus, there is a need to change the definition of response to the pharmacologic treatment of HRS. Full response will be defined by return of $\mathrm{sCr}$ to a value within $0.3 \mathrm{mg} / \mathrm{dL}(26.5 \mu \mathrm{mol} / \mathrm{L})$ of the baseline value. Partial responses will be defined by a regression of at least one AKI stage with a fall in the $\mathrm{sCr}$ value to $\geq 0.3 \mathrm{mg} / \mathrm{dL}$ $(26.5 \mu \mathrm{mol} / \mathrm{L})$ above the baseline value. Nevertheless, we should recognise that preliminary data suggest that even a partial decrease of $\mathrm{sCr}$ from baseline may be associated with improved short term survival, irrespective of whether or not the patient achieves HRS reversal $(\mathrm{sCr}$ $<1.5 \mathrm{mg} / \mathrm{dL}$ ). ${ }^{38}$ These data suggest that the degree of improvement in $\mathrm{SCr}$ may be more relevant than achieving a finite level of renal function. 


\section{CONCLUSIONS AND FUTURE PERSPECTIVES}

Based on the most recent studies on AKI in patients with cirrhosis and ascites, a new algorithm for the management of AKI in these patients is proposed for clinical practice and for future research. The main innovative aspects of this new algorithm are the following:

- The adoption of the main point derived from the application of the KDIGO criteria in the definition of AKI in patients with cirrhosis, namely, use of dynamic changes of $\mathrm{sCr}$

- A more structured diagnostic process, in order to allow a rational application of the therapeutic resources, avoiding potentially undesirable consequences of overtreatment of AKI as a result of indiscriminant use of KDIGO criteria

- The definitive removal of any cut-off value of sCr from the criteria for diagnosis of HRS in the setting of AKI, but maintaining the remaining previous criteria (box 1).

Several issues remain to be addressed: (1) the impact of the management of AKI according to the new algorithm on the outcome of these patients should be tested in future prospective studies; and (2) the role of the new biomarkers of renal tubular damage in predicting the progression and prognosis of AKI, and in the differential diagnosis of the different types of AKI. ${ }^{36} 37$

In summary, the results of the latest consensus conference of the ICA introduces a new dynamic definition of AKI in patients with cirrhosis, on which a new treatment algorithm is based, representing a substantial change from the traditional criteria used until now in the definition of AKI and type 1 HRS.

Contributors Organisation of the meeting: PA; Analysis of data from the literature: $\mathrm{PA}, \mathrm{PG}, \mathrm{FW}, \mathrm{MB}$, $T D B, A G, R M, R J, S K S, S P, K M$, SSL, FD, FS, PC, WRK, VA, GG; Drafting and writing the manuscript: PA, PG, FW, MB, SP, GG; Critical revision of data and manuscript revision: $P A, P G, F W, M B, T D B, A G, R M$, $R J, S K S, S P, K M, S S L, F D, F S, P C, V A, G G$.

Funding PG: Research funding from Sequana Medical, Grifols SA; Consultancy Advisor to Ferring Pharmaceuticals; Competitive Public Grant Funding from: Fondos de Investigación Instituto de Salud CarlosIII (FIS12/0330) and Agència de Gestió d'Ajuts Universitaris i de Recerca (2014 SGR 708). MB: Consultant to CSL Behring GmbH, Baxter Healthcare $\mathrm{SA}$; speaker to Behring $\mathrm{GmbH}$, Baxter Healthcare SA, PPTA Europe. AG: Consultant to CSL Behring. RJ: Consultant to Ocera Therapeutics Inc, Conatus Pharmaceuticals Inc; Research grant from: Grifols Inc, Gambro AB, Sequana Medical AG, Norgine BV, Ocera Therapeutics Inc (the latter five companies are all involved in research collaboration): Speaker to Grifols Inc; Norgine BV; Inventors of Ornithine Phenylacetate licensed by UCL to Ocera Therapeutics; UCL spinout Yaqrit which will in-licence five of the following inventions in which $\mathrm{R}$ Jalan is the main inventor: YAQ001 nanoporous carbons for prevention of gut bacterial translocation, UCL liver dialysis device, DASIMAR biomarker for liver failure, Neutrophil for test: biomarker for predicting infection in decompensated cirrhotic patients, Urinary toll-like receptor 4: for differential diagnosis of renal dysfunction of cirrhosis. SSL: Consultant to Ikaria and Grifols. PC: Lecturer to Baxter Healthcare SA, Kedrion, Grifols. VA: Received grant and research support from Grifols.

Competing interests None.

Provenance and peer review Not commissioned; externally peer reviewed.

Editors' note This article is being published jointly in Gut and Journal of Hepatology.

(C) 2015 BMJ Publishing Group Ltd, British Society of Gastroenterology and European Association for the Study of the Liver.
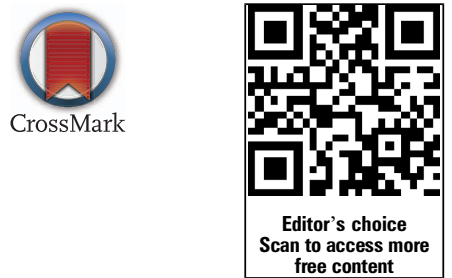

To cite Angeli $P$, Gines $P$, Wong $F$, et al. Gut 2015;64:531-537

Received 20 November 2014

Revised 4 December 2014

Accepted 11 December 2014

Published Online First 28 January 2015

Gut 2015;64:531-537.

doi:10.1136/gutjnl-2014-308874

\section{REFERENCES}

1 Arroyo V, Gines P, Gerbes AL, et al. Definition and diagnostic criteria of refractory ascites and hepatorenal syndrome in cirrhosis. Hepatology 1996;23:164-76.

2 Salerno F, Gerbes A, Gines P, et al. Diagnosis, prevention and treatment of the hepatorenal syndrome in cirrhosis: a consensus workshop of the International Ascites Club. Gut 2007;56 1310-18.

3 Wong F, Nadim MK, Kellum JA, et al. Working party proposal for a revised classification system of renal dysfunction in patients with cirrhosis. Gut 2011;60:702-9.

4 Angeli P, Sanyal A, Moller S, et al. Current limits and future challenges in the management of renal dysfunction in patients with cirrhosis: report from the International Club of Ascites. Liver Int

2013;33:16-23.

5 Bellomo R, Ronco C, Kellum J, et al. Acute renal failure-definition, outcome measures, animal models, fluid therapy and information technology needs: The Second International Consensus Conference of the Acute Dialysis Quality Initiative (ADQI) Group. Crit Care 2004:8:R204-12.

6 Mehta RL, Kellum JA, Shah SV, et al. Acute Kidney Injury Network: report of an initiative to improve outcomes in acute kidney injury. Crit Care 2007;11: R31.

7 Kidney Disease: Improving Global Outcomes (KDIGO) Acute Kidney Injury Work Group. KDIGO Clinical Practice Guideline for Acute Kidney Injury. Kidney Int 2012;2(Suppl):1-138

8 Belcher JM, Garcia-Tsao G, Sanyal AJ, et al. Association of AKI with mortality and complications in hospitalized patients with cirrhosis. Hepatology 2013;57:753-62.
9 Piano S, Rosi S, Maresio G, et al. Evaluation of the Acute Kidney Injury Network criteria in hospitalized patients with cirrhosis and ascites. I Hepatol 2013;59:482-9.

10 Fagundes C, Barreto R, Guevara M, et al. A modified acute kidney injury classification for diagnosis and risk stratification of impairment of kidney function in cirrhosis. J Hepatol 2013;59:474-81.

11 Tsien CD, Rabie R, Wong F. Acute kidney injury in decompensated cirrhosis. Gut 2013;62:131-7.

12 de Carvalho JR, Villela-Nogueira CA, Luiz RR, et al. Acute kidney injury network criteria as a predictor of hospital mortality in cirrhotic patients with ascites. J Clin Gastroenterol 2012;46:e21-6.

13 Wong F, O'Leary JG, Reddy KR, et al. New consensus definition of acute kidney injury accurately predicts 30-day mortality in patients with cirrhosis and infection. Gastroenterology 2013;145:1280-8.

14 Altamirano J, Fagundes C, Dominguez $\mathrm{M}$, et al. Acute kidney injury is an early predictor of mortality for patients with alcoholic hepatitis. Clin Gastroenterol Hepatol 2012:10:65-71.

15 Angeli $P$, Rodríguez E, Piano S, et al. Acute kidney injury and acute-on-chronic liver failure classifications in prognosis assessment of patients with acute decompensation of cirrhosis. Gut Published Online First: 13 Oct 2014. doi:10.1136/gutjnl-2014-307526

16 Sherman DS, Fish DN, Teitelbaum I. Assessing renal function in cirrhotic patients: problems and pitfalls. Am J Kidney Dis 2003:41:269-78.

17 Caregaro L, Menon F, Angeli $P$, et al. Limitations of serum creatinine level and creatinine clearance as filtration markers in cirrhosis. Arch Intern Med 1994;154:201-5.

18 Spencer K. Analytical reviews in clinical biochemistry: the estimation of creatinine. Ann Clin Biochem 1986:23(Pt 1):1-25.

19 Bataller $R$, Ginès $P$, Guevara $M$, et al. Hepatorenal syndrome. Semin Liver Dis 1997:17:233-47.

20 Ginès $P$, Arroyo $V$, Vargas $V$, et al. Paracentesis with intravenous infusion of albumin as compared with peritoneovenous shunting in cirrhosis with refractory ascites. N Engl J Med 1991;325:829-35.

21 Sort $\mathrm{P}$, Navasa M, Arroyo $\mathrm{V}$, et al. Effect of intravenous albumin on renal impairment and mortality in patients with cirrhosis and spontaneous bacterial peritonitis. N Engl J Med 1999;341: 403-9.

22 Ginès A, Fernández-Esparrach G, Monescillo $A$, et al. Randomized trial comparing albumin, dextran 70 , and polygeline in cirrhotic patients with ascites treated by paracentesis. Gastroenterology 1996:111:1002-10.

23 Angeli $P$, Fasolato S, Mazza E, et al. Combined versus sequential diuretic treatment of ascites in non-azotaemic patients with cirrhosis: results of an open randomized clinical trial. Gut 2010:59:98-104.

24 Angeli P, Gatta A, Caregaro L, et al. Tubular site of renal sodium retention in ascitic liver cirrhosis evaluated by lithium clearance. Eur J Clin Invest 1990;20:111-17.

25 Joannidis M, Metnitz B, Bauer $\mathrm{P}$, et al. Acute kidney injury in critically ill patients classified by AKIN versus RIFLE using the SAPS 3 database. Intensive Care Med 2009;35:1692-702.

26 Tu KH, Jenq CC, Tsai MH, et al. Outcome scoring systems for short-term prognosis in critically ill cirrhotic patients. Shock 2011:36:445-50.

27 Wong F, O'Leary JG, Reddy R, et al. A cut-off serum creatinine value of $1.5 \mathrm{mg} / \mathrm{dL}$ for $A K L$ - to be or not to be. J Hepatol 2014. doi:10.1016/j.jhep.2014.10.047

28 Arroyo V. Acute kidney injury (AKI) in cirrhosis: should we change current definition and diagnostic criteria of renal failure in cirrhosis? J Hepatol 2013:59:415-17.

29 Bagshaw SM, Uchino S, Cruz D, et al. A comparison of observed versus estimated baseline creatinine for determination of RIFLE class in patients with acute 
kidney injury. Nephrol Dial Transplant 2009;24: 2739-44.

30 Lisman T, van Leeuwen Y, Adelmeijer J, et al. Interlaboratory variability in assessment of the model of end-stage liver disease score. Liver Int 2008;28: 1344-51.

31 Francoz C, Prié D, Abdelrazek W, et al. Inaccuracies of creatinine and creatinine-based equations in candidates for liver transplantation with low creatinine: impact on the model for end-stage liver disease score. Liver Transp/ 2010;16:1169-77.

32 Boyer TD, Medicis JJ, Pappas SC, et al. A randomized, placebo-controlled, double-blind study to confirm the reversal of hepatorenal syndrome type 1 with terlipressin: the REVERSE trial design. Open Access I Clinical Trials 2012;4:39-49.

33 Boyer TD, Sanyal AJ, Garcia-Tsao G, et al. Predictors of response to terlipressin plus albumin in hepatorenal syndrome (HRS) type 1: relationship of serum creatinine to hemodynamics. J Hepatol 2011;55:315-21.

34 Rodriguez E, Elia C, Solà E, et al. Terlipressin and albumin for type-1 hepatorenal syndrome associated with sepsis. J Hepatol 2014;60:955-61.

35 Trawale JM, Paradis V, Rautou PE, et al. The spectrum of renal lesions in patients with cirrhosis: a clinicopathological study. Liver Int 2010;30:725-32.

36 Fagundes C, Pépin MN, Guevara M, et al. Urinary neutrophil gelatinase-associated lipocalin as biomarker in the differential diagnosis of impairment of kidney function in cirrhosis. J Hepatol 2012;57:267-73.

37 Belcher JM, Sanyal AJ, Peixoto AJ, et al. Kidney biomarkers and differential diagnosis of patients with cirrhosis and acute kidney injury. Hepatology 2014;60:622-32.

38 Boyer TD, Sanyal AJ, Wong $F$, et al. Initial report of a large, randomized, double blind, placebo-controlled, phase 3 trial of terlipressin plus albumin for the treatment of type 1 hepatorenal syndrome (HRS-1): the REVERSE study. Hepatology 2014;60:255A. (Abstract). 